\title{
Der Staat als Aktionär der letzten Instanz: zum krisenbedingten Wandel der Staatsaufgaben
}

von Joachim Wieland

Der Staat ist im Gefolge der Finanzkrise zum Bankier wider Willen geworden. Wie soll er sich in dieser Rolle verhalten? Soll er das Gemeinwohl - Bekämpfung einer Kreditklemme, Verhinderung risikoerhöhender Boni - auch in der Unternehmenspolitik fördern? Oder soll er seine Beteiligungen so schnell wie möglich wieder privatisieren - auf die Gefahr hin, in der nächsten Krise erneut als Aktionär der letzten Instanz zum Einsatz öffentlicher Gelder gezwungen zu werden? Der Beitrag zeigt vor dem Hintergrund geschichtlicher Erfahrungen und ausländischer Entwicklungen die Handlungsoptionen innerhalb der rechtlichen Rahmenbedingungen auf und plädiert gegen einen Rückzug auf die Position des Aktionärs der letzten Instanz.

As a consequence of the global financial crisis the state has become a banker against its will. How shall the state act in this role? Should it fight a credit crunch and risky bonuses for top employees in the interest of the common good? Or shall it sell its shares as soon as possible running the risk to be forced to spend huge amounts of the taxpayers' money during the next crisis? The author shows the state's options against the background of historical experiences in Germany and international developments considering the legal framework and argues against a retreat to the position of a shareholder of last instance.

„Möglicherweise werden wir am Ende akzeptieren müssen, dass der Staat in systemischen Bankenkrisen der Aktionär der letzten Instanz bleibt.“1

\section{Das Problem}

Das Verhältnis des Staates zur Wirtschaft hat sich als Folge der Finanz- und Wirtschaftskrise grundlegend verändert. Vor der Krise schien es so, als sei der Staat nach vielen Privatisierungsschritten auf dem Wege, nicht nur die eigene wirtschaftliche Betätigung aufzugeben, sondern auch immer mehr zuvor als

1 Ackermann, J.: Zukunft des Bankgeschäfts. Zwischen neuen Marktstrukturen und veränderter Regulierung, Eröffnung der Euro Finance Week, Frankfurt/M., 16.11.2009, 9, online unter www.db.com/ presse/de/downloads/EuroFinanceWeek_Eroeffnung_JA.pdf. 
Verwaltungsaufgaben wahrgenommene Aufgaben Privaten zu überlassen. Die Zeiten, in denen der Staat sich selbst unternehmerisch betätigte, schienen der Vergangenheit anzugehören. Die Finanzkrise, die 2007 in den USA ihren Anfang nahm und dann schnell die ganze Welt erfasste und sich zur Wirtschaftskrise ausweitete, nötigte nicht nur in Deutschland, sondern auch in von ihrer Wirtschaftsordnung vergleichbaren Staaten wie Großbritannien und den USA die politisch Verantwortlichen, massiv in das Wirtschaftsgeschehen einzugreifen. Schienen zunächst noch die Übernahme von Bürgschaften und die Gewährung von Garantien des Staates für privatwirtschaftliche Unternehmen auszureichen, stellte sich bald heraus, dass oft nur die teilweise oder sogar vollständige Verstaatlichung von Unternehmen einen Ausweg aus der Krise zu weisen. Das zeigte sich zunächst bei Banken, die als systemrelevant für das Funktionieren der nationalen und internationalen Finanzordnung eingestuft wurden, machte dort aber nicht Halt. Vielmehr sahen sich mehrere Staaten genötigt, auch Unternehmen außerhalb des Bankensektors zu übernehmen. In Deutschland ist das beste Beispiel dafür die Adam Opel AG in Rüsselsheim, die zu 70 \% vom Bund übernommen wurde. Für den Staat wirft diese Entwicklung die Frage auf, wie er seiner Rolle als Gesellschafter privatwirtschaftlich handelnder Unternehmen, die er nicht angestrebt hat, sondern die ihm gegen seine Intentionen zugefallen ist, wahrnehmen soll. Soll er sich als stiller Gesellschafter von allen unternehmerischen Entscheidungen fernhalten und auf die Manager vertrauen, die in seinen Unternehmen tätig sind? Oder ist es angezeigt bzw. sogar geboten, seine umfassende Aufgabe der Förderung des Allgemeinwohls auch in seiner Funktion als Gesellschafter eines privatwirtschaftlichen Unternehmens wahrzunehmen?

Die praktischen Konsequenzen zeigen sich besonders deutlich in der öffentlichen Auseinandersetzung um die befürchtete Kreditklemme, um die Neuordnung der Landesbanken und um die Begrenzung der Vergütung von Managern. Soll oder muss der Staat auf eine Begrenzung von Boni dort hinwirken, wo er als Gesellschafter in von ihm beherrschten oder mitbeherrschten Unternehmen insbesondere des Finanzsektors Einfluss nehmen kann, wenn er mit der wohl herrschenden Auffassung bei den Ökonomen in verfehlten Anreizsystemen bei der Vergütung eine Ursache für die Krise sieht? Oder soll er auch in den Banken und Unternehmen, in denen er als Gesellschafter über Einfluss verfügt, von gestaltender Einflussnahme auf das privatwirtschaftliche Handeln Abstand nehmen, weil es nicht seine Aufgabe ist, sich wirtschaftlich zu betätigen? Eine vergleichbare Frage stellt sich bezüglich der Gewährung von Krediten. Der Staat als Gesellschafter einer Bank kann seinen Einfluss dahin ausüben, dass Kredite bereitwil- 
liger gewährt werden, wenn ihm das volkswirtschaftlich sinnvoll erscheint. Betriebswirtschaftliche Risikoerwägungen, die in anderen Banken offenbar für eine Zurückhaltung bei der Kreditvergabe sorgen, müssten dann zurückstehen. Schließlich fällt die Organisation von Landesbanken nicht in die Zuständigkeit des Bundes, sondern in die der Länder, soweit es um gesetzliche Regelungen geht. Wenn aber der Bund als Gesellschafter handelt, ist er nicht an Kompetenzgrenzen gebunden, sondern kann kraft seines Anteilseigentums tätig werden. Solange der Staat nach eigenem Gutdünken und nach den wirtschaftspolitischen Grundüberzeugungen der handelnden Politiker entscheiden konnte, ob er sich an privaten Unternehmen beteiligen oder das Handeln der öffentlichen Verwaltung privatisieren wollte, konnte er dieser Entscheidungssituation entgehen, indem er eine Gesellschafterstellung nicht begründete oder vorhandene Unternehmensbeteiligungen wieder veräußerte.

Diese Entscheidungsfreiheit besteht nach dem Ausbruch der Finanz- und Wirtschaftskrise jedenfalls nur noch begrenzt. Zumindest für systemrelevante Banken gilt nicht nur gegenwärtig, sondern auch in Zukunft, dass der Staat Aktionär der letzten Instanz bleiben wird. Er kann sich dieser Stellung zwar nach der Überwindung der Krise entziehen, muss aber stets damit rechnen, bei Ausbruch einer neuen Krise wieder als Aktionär in die Pflicht genommen zu werden. Das verändert die wirtschaftliche Ordnung grundlegender, als das bislang weithin wahrgenommen worden ist. Auf der Seite der betroffenen Finanzinstitute haben sich die Rahmenbedingungen insoweit fundamental verändert, als sie dem Risiko des Scheiterns enthoben sind, dass in einer Marktwirtschaft für jeden Marktteilnehmer selbstverständlich sein sollte. Die Freistellung vom Insolvenzrisiko begünstigt die Neigung zum moral hazard. Der Anreiz risikoreiche, kurzfristig aber lukrative Geschäfte einzugehen, wächst beträchtlich, weil das in vielen Banken übliche Bonussystem den sich schnell einstellenden wirtschaftlichen Erfolg prämiert, der Staat als Aktionär in Krisensituationen aber faktisch gezwungen ist, systemrelevante Banken mit Mitteln aus öffentlichen Kassen zu stützen. Verhält sich der Staat in dieser Situation passiv, riskiert er, den moral hazard indirekt zu unterstützen. Übt er seine Gesellschafterstellung aus, setzt er sich dem Vorwurf aus, sich auf Tätigkeitsfelder $\mathrm{zu}$ begeben, die nicht $\mathrm{zu}$ den eigentlichen Staatsaufgaben gehören und für die seine Amtsträger nicht über die nötige Ausbildung und Erfahrung verfügen. Die Bezeichnung des Staates als „Agentur der 
schlechten Risiken“2 gewinnt in dieser Perspektive eine ganz neue Bedeutung: Als Aktionär letzter Instanz ist der Staat nur in der Krise gefordert. Wenn es systemrelevanten Banken wirtschaftlich gut geht und sie Gewinne erzielen, kann auf den Staat als Gesellschaft aus Sicht der Wirtschaft gut verzichtet werden. Kann es sich aber der Staat im Interesse des Gemeinwohls erlauben, nur als Aktionär der letzten Instanz tätig zu werden, die Geschäftspolitik systemrelevanter Banken aber weder in der Krise - in der er zum Gesellschafter wird - noch in krisenfreien Zeiten - in denen er auf seine Gesellschafterstellung verzichten soll - inhaltlich zu beeinflussen?

An dieser Problemstellung ändert auch der von Bankenkreisen angeregte europäische Notfallfonds nicht, der von Banken und Staaten finanziert werden soll. Mit dem Fonds soll sich „eine Lastenteilung zwischen Finanzindustrie und der öffentlichen Hand besser erreichen“ lassen, „wenn und insoweit der Fonds von beiden gespeist würde “3. Ein solcher Fonds wäre aus Sicht der Banken sicherlich attraktiv, weil die für eine Krisenintervention erforderlichen Mittel bereits im Vorhinein zur Verfügung stünden. Tatsächlich ließen sich vielleicht „,mitternächtliche Rettungsaktionen mit all ihren Problemen vermeiden“4. Ob sich aber die Zugangsbedingungen zu dem Fonds ex ante so festlegen ließen, dass „Unsicherheit und moral hazard begrenzt würden“"5, wie es der Vorstandsvorsitzende der Deutschen Bank annimmt, muss bezweifelt werden. Gerade weil ein Fonds mehr Sicherheit und formale Regeln für die Krisenhilfe mit Staatsmitteln schaffen würde, während unter den gegebenen Umständen eine Wiederholung der Verweigerung staatlicher Hilfe wie im Falle Lehmann Brothers nie völlig auszuschließen ist, würde moral hazard verstärkt werden. Der Fonds wäre nicht nur Sinnbild der zu erwartenden Staatshilfe, sondern würde diese rechtlich institutionalisieren und mit den Weihen eines Rechtsanspruchs auf finanzielle Unterstützung versehen. Das Problem für den Staat als Aktionär bliebe auch bei Errichtung eines Notfallfonds bestehen - nur wäre seine Aktionärsstellung durch den Fonds vermittelt. Wie aber ist das Problem zu lösen?

Um diese Frage zu beantworten, wird in der Folge zunächst ein kurzer Blick auf die gerade in Deutschland wechselvolle Geschichte der Einwirkung des Staates

2 Löwer, W.: Der Staat als Wirtschaftssubjekt und Auftraggeber, in: Veröffentlichungen der Vereinigung der Deutschen Staatsrechtslehrer, 60 (2001), 416-452, hier 436.

3 Ackermann, J., a. a. O., 9.

4 Ebd.

5 Ebd. 
auf die Privatwirtschaft gegeben (II.), bevor das Staathandeln in der aktuellen Wirtschaftskrise in den Blick genommen wird (III.). Ausländische Erfahrungen können ebenfalls zur Problemlösung beitragen (IV.). Werden dann noch die Handlungsalternativen (V.) und die normativen Rahmenbedingungen analysiert (VI.), lassen sich Folgerungen ziehen (VII.).

\section{Die geschichtliche Entwicklung}

Die Beteiligung des Staates an volkswirtschaftlich wichtigen Unternehmen hat in Deutschland Tradition. Je nach vorherrschenden wirtschaftpolitischen und wirtschaftstheoretischen Auffassungen haben Umfang und Intensität der Unternehmensbeteiligungen jedoch erheblich geschwankt. Vor dem Ersten Weltkrieg kam das paradigmatisch im Bergbau zum Ausdruck, der seinerzeit eine Schlüsselindustrie bildete. Der Kameralismus des 18. Jahrhunderts war davon ausgegangen, dass die Bodenschätze als natürliche Ressourcen die wirtschaftliche Autonomie eines Staates sicherten. Dementsprechend wurde der Bergbau umfassender staatlicher Direktion durch die Bergämter unterworfen, die auch die Preise regulierten. ${ }^{6}$ Nachdem sich der Wirtschaftsliberalismus im deutschen Bergbau nur mühsam und langsam durchgesetzt hatte, überließ der Staat erst in der zweiten Hälfte des 19. Jahrhunderts auf der Grundlage der nunmehr eingeräumten Bergbaufreiheit privaten Unternehmen die selbständige Leitung ihrer Gruben und beschränkte sein eigenes Handeln im Wesentlichen auf die Gefahrenabwehr. ${ }^{7}$ Nach der Jahrhundertwende musste die Bergbaufreiheit dann wieder Staatsvorbehalten weichen. Das Vertrauen auf die Selbstregulierung des Marktes hatte zu so starken Konzentrationsbewegungen und Monopolisierungstendenzen in der Bergwirtschaft geführt, dass ein Eingreifen des Staates sich als unumgänglich erwies. Die Monopolisierung von Rohstoffen, auf deren Nutzung die Volkswirtschaft angewiesen war, brachte die Gefahr von Versorgungsengpässen und überhöhten Preisen mit sich, zumal die großen Bergwerksgesellschaften unliebsamen Wettbewerb durch Demarkationsverträge verhinderten. Nachdem Preußen sich zunächst vergeblich bemüht hatte, die Entwicklung des Bergbaus mit marktkonformen Mitteln zu beeinflussen, wurde 1907 ein Staatsvorbehalt eingeführt, der

6 Die Direktionsbefugnisse des Staates kommen beispielhaft in den einschlägigen Regelungen in Teil II, 16, Abschnitt 4 des Preußischen Allgemeinen Landrechts zum Ausdruck, der das gemeine deutsche Bergrecht gegen Ende des 18. Jahrhunderts widerspiegelte; näher dazu Wieland, J.: Die Konzessionsabgaben, Berlin, 1991, $61 \mathrm{ff}$.

7 Siehe die Regelungen des Allgemeinen Berggesetzes von 1865 in Preußen, GS S. 705; dazu Wieland, J., a. a. O., $66 \mathrm{ff}$. 
allein dem Staat das Recht einräumte, Steinkohle und andere wichtige Bodenschätze zu gewinnen. ${ }^{8}$ Auch die Eisenbahnen wurden verstaatlicht. ${ }^{9}$

Von 1914 bis 1918 führte der Übergang zur Kriegswirtschaft zu einer halb staatlichen Lenkung der Rüstungsproduktion. So genannte „Kriegsrohstoffgesellschaften" in der Rechtsform von Aktiengesellschaften standen unter staatlicher Aufsicht, die ein Vetorecht umfasste. Die dringend benötigte Aluminium- und Stickstoffindustrie baute der Staat in eigener Regie auf. ${ }^{10}$ Bürgschaften der Regierung deckten die notwendigen Investitionen ab. Die Regierung übertrug den „staatlich-privaten Zwittergesellschaften“ in diesem Wirtschaftssystem des ,intensivierten Korporatismus“ ein Stück „öffentlicher Herrschaftsgewalt“. In dieser Wirtschaftsordnung konnten private Unternehmer sehr große Gewinne erzielen, solange sie die Wünsche der Staatsleitung nach ausreichender Rüstungsproduktion erfüllten. ${ }^{11}$

In der Weimarer Republik setzte sich die verstärkte wirtschaftliche Betätigung des Staates fort. Die Verkehrsunternehmen des Reichs beschäftigten fast 1,2 Mio. Menschen und erwirtschafteten etwa 1929 einen Überschuss von 1,1 Mrd. Reichsmark, der in etwa der Summe der Dividenden aller deutschen Aktiengesellschaften in diesem Jahr entsprach. Das Reich besaß mit der Elektrowerke AG das größte Elektrizitätsunternehmen Deutschlands und hatte ein faktisches Monopol in der Aluminiumproduktion sowie eine beachtliche Beteiligung an der Stickstoffproduktion sowie an metallverarbeitenden Unternehmen. ${ }^{12} 1923$ führte das Reich seine industriellen Beteiligungen in den Vereinigten Industrieunternehmungen AG (Viag) zusammen, die nicht länger für die Rüstung, sondern seit dem Kriegsende für den zivilen Bedarf produzierten. Der Konzern Viag umfasste Unternehmen aus den Wirtschaftsbereichen Elektrizität, Eisen- und Maschinenbau, Aluminium, Kalkstickstoff, Bankwesen und Industrieanlagen. Das parallele Vorgehen Preußens mündete 1926 in die Gründung der Preußischen Bergwerksund Hütten-AG (Preußag) sowie 1927 in die Preußische Elektrizitätsaktiengesellschaft (Preußenelektra). Beide wurden 1929 mit der Beteiligung an der Bergwerks-AG Recklinghausen und der Hibernia zur Vereinigte Elektrizitäts-

8 Preußisches Gesetz vom 18.06. 1907, GS S. 119; dazu Wieland, J., a. a. O., 72 ff.

9 Ronellenfitsch, M.: Wirtschaftliche Betätigung des Staates, in: Isensee, J./Kirchhof, P. (Hg.): Handbuch des Staatsrechts der Bundesrepublik Deutschland, Band IV, 3. Aufl., Heidelberg, 2006, § 98 Rn. 10.

10 Ebd., § 98 Rn. 11.

11 Zum Ganzen Wehler, H.-U.: Deutsche Gesellschaftsgeschichte, Bd. 4, Vom Beginn des Ersten Weltkriegs bis zur Gründung der beiden deutschen Staaten 1914-1949, München, 2003, $47 \mathrm{ff}$.

12 Ambrosius, G.: Der Staat als Unternehmer, Göttingen, 1984, $64 \mathrm{ff}$. 
und Bergwerks-AG (VEBA) verschmolzen. ${ }^{13}$ Auch die Wirtschaftsordnung der Weimarer Republik war also durch einen großen öffentlichen Sektor der Wirtschaft und einen „staatsnahen Korporativismus“ mit einer „Interessenverschränkung zwischen Großindustrie und Ministerialbürokratie" gekennzeichnet. Der staatliche Interventionismus führte zu einer ,gelenkten Marktwirtschaft“. Das zeigte sich besonders deutlich in der Weltwirtschaftskrise. Nachdem am 13. Juli 1931 die zweitgrößte deutsche Bank, die Darmstädter und Nationalbank (Danat) zusammengebrochen war, übernahm der Staat am folgenden Tag $75 \%$ der Aktien der Dresdner Bank, die ebenfalls vor dem Bankrott zu stehen schien. 1932 wurde die Dresdner Bank auf der Grundlage einer Notverordnung des Reichspräsidenten $^{14}$, die der Reichsregierung zur Sanierung die Beteiligung an Banken gestattete, verstaatlicht. An der Commerzbank erwarb der Staat $70 \%$ des Gesellschaftskapitals. ${ }^{15}$ Zur Sanierung der Danat trug der Staat bei, indem er Industriellen aus dem Ruhrgebiet das für den Kauf von Bankaktien benötigte Geld lieh. ${ }^{16}$ Die systemische Krise des Finanzsystems zwang also schon während der Weltwirtschaftskrise den Staat dazu, Aktionär notleidender Banken zu werden.

Während der Zeit des Nationalsozialismus verstärkten sich die staatlichen Einwirkungen auf die Wirtschaft dann noch einmal erheblich über das Maß hinaus, das sie in der Weimarer Republik und auch im Ersten Weltkrieg erreicht hatten. Die IG Farben wurde zum Teil verstaatlicht. Das Volkswagenwerk in Wolfsburg und die Reichswerke Hermann Göring im Juli 1937 in Salzgitter wurden gleich als reine Staatskonzerne im Rahmen des Vierjahresplans gegründet. In die Reichswerke wurden nach dem Anschluss Österreichs 30 Industriebetriebe eingegliedert. Tschechische und polnische Betriebe wurden zu Gunsten des Staates zwangsenteignet. Als Leiter des Vierjahresplanamtes und Reichsbeauftragter für Rohstoff- und Devisenfragen hatte Göring vielfältige Einflussmöglichkeiten auf die deutsche Volkswirtschaft, die seit 1936 zur Kriegswirtschaft umgebaut wurde. Im Ergebnis waren Staat und Wirtschaft zwischen 1933 und 1945 eng verschränkt. ${ }^{17}$

13 Ronellenfitsch, M., a. a. O., § 98 Rn. $12 \mathrm{f}$.

$14 \S 1$ Buchst. a Verordnung des Reichspräsidenten über die Sanierung von Bankunternehmen vom 20.02. 1932, RGBl. 1932 I, 83.

15 Commerzbank - Aus der Geschichte einer fast 140-jährigen Großbank, 2 f., online unter https://www. commerzbank.de/media/konzern/geschichte/CB-Geschichte_2009.pdf.

16 Wehler, H.-U., a. a. O., 262 und $268 \mathrm{ff}$.

17 Zum Ganzen ebd., $691 \mathrm{ff}$. 
Trotz der Teilung Deutschlands war der Bund nach 1949 in deutlich größerem Umfang wirtschaftlich tätig als das Reich in der Weimarer Republik. Zwischen 1960 und 1982 erwarb der Bund im Jahresdurchschnitt mehr als 39 Beteiligungen an Wirtschaftsunternehmen. Den Schwerpunkt der Unternehmen in staatlicher Hand bildeten die Viag, die Saarbergwerke AG, die IndustrieverwaltungsGmbH, die Veba AG mit einem Bundesanteil von 43,8 \% und das Volkswagenwerk, an dem der Bund zu $20 \%$ beteiligt war. Auch die Deutsche Lufthansa AG gehörte dem Staat. Um 1980 arbeiteten ungefähr 1,3 Mio. Menschen in Unternehmen des Bundes. ${ }^{18}$ Mit dem Regierungswechsel 1982 setzte dann eine Politik der Privatisierung ein, in deren Rahmen sich der Bund von vielen seiner Unternehmen trennte. Allerdings wurde es schon bald als offene Frage bezeichnet, ob diese Privatisierungsmaßnahmen das Anwachsen wirtschaftlicher Staatstätigkeit nachhaltig und auf Dauer aufhalten könnten. ${ }^{19}$ Die Erfahrungen in der aktuellen Finanz- und Wirtschaftskrise zeigen die Berechtigung der Frage.

\section{Die Finanz- und Wirtschaftskrise}

Die Finanzkrise, die Deutschland 2007 erreichte, hatte ihren Ursprung in der subprime-Krise in den USA. Dort hatten Banken Immobilienkredite an nicht hinreichend zahlungskräftige Schuldner vergeben und anschließend verbrieft an andere Banken weiter verkauft. Als die Spekulationsblase auf dem US-Immobilienmarkt zusammenbrach und in der Folge die zuvor überaus stark gestiegenen Immobilienpreise in großem Umfang fielen, konnten viele Kredite nicht mehr bedient werden. Dadurch wurde das Vertrauen in die Werthaltigkeit der Verbriefungen nachhaltig erschüttert. Deshalb mussten auch deutsche Banken Abschreibungen in Milliardenhöhe vornehmen. Die Commerzbank war besonders stark betroffen, weil sie sich 2008 gegenüber der Allianz SE zu einer Fusion mit deren Tochter Dresdner Bank verpflichtet hatte, die ebenfalls Abschreibungen in Milliardenhöhe vorzunehmen hatte. Diese Fusion wurde im Januar 2009 vollzogen. Sie verschärfte die finanziellen Schwierigkeiten der Commerzbank beträchtlich. Bereits am 19. Dezember 2008 vereinbarte sie deshalb mit dem Sonderfonds Finanzmarktstabilisierung (SoFFin) ein Kreditprogramm für den Mittelstand. ${ }^{20}$ Im Rahmen dieser Vereinbarung stellte die Commerzbank zusätz-

18 Ambrosius, G., a.a.O., $116 \mathrm{f}$.

19 Ronellenfitsch, M., a. a. O., §98 Rn. 18.

20 Siehe die Pressemitteilung der Commerzbank AG vom 19.12. 2008. Die Pressemitteilungen der Commerzbank AG sind abrufbar unter https://www.commerzbank.de/de/hauptnavigation/presse/archiv_ presse_mitteilungen/2009/quartal_09_04/presse_archiv_09_04.html. 
lich 2,5 Mrd. € für Mittelstandskredite zur Verfügung. Die Vereinbarung sah im Gegenzug eine stille Beteiligung des SoFFin an der Commerzbank in Höhe von 8,2 Mrd. $€$ vor, die mit $9 \%$ jährlich verzinst wird. Außerdem gewährte der SoFFin eine Garantie für Schuldverschreibungen über bis zu $15 \mathrm{Mrd}$ €. Die Commerzbank verpflichtete sich, die Gehälter ihrer Vorstände auf $500.000 € \mathrm{zu}$ begrenzen. ${ }^{21}$ Noch im Januar 2009 leistete der SoFFin eine weitere stille Einlage in Höhe von 8,2 Mrd. €. ${ }^{22}$ Gemäß einem Beschluss der Hauptversammlung der Commerzbank am 15. und 16. Mai zeichnete der SoFFin gut 295 Mio. neue Inhaberaktien der Bank. ${ }^{23}$ Die Kapitalerhöhung gegen Bareinlage unter Ausschluss des Bezugsrechts der Aktionäre (gem. § 7 Finanzmarktstabilisierungsgesetz) führte dazu, dass der Bund nunmehr mit $25 \%$ des Stammkapitals plus einer Aktie größter Aktionär der fusionierten Bank $^{24}$ ist und über eine aktienrechtliche Sperrminorität ${ }^{25}$ verfügt.

Mittlerweile vollständig in der Hand des Staates ist die HypoRealEstate (HRE). Die HRE hatte keine eigene Einlegerbasis, sondern refinanzierte die von ihr vergebenen Kredite, indem sie ihrerseits Kredite bei Banken aufnahm, die über ein großes Einlagengeschäft verfügten. Ihre Tochter Deutsche Pfandbriefbank (Depfa) in Dublin hatte sich darauf spezialisiert, langfristige Staatsanleihen mit kurzfristigen Mitteln anderer Kreditinstitute zu finanzieren. ${ }^{26}$ Nach dem Zusammenbruch der amerikanischen Bank Lehmann Brothers am 15. September 2008 führte die Erkenntnis, dass nicht automatisch mit einer Rettung insolventer Finanzunternehmen durch die Staaten gerechnet werden konnte, zu einem großen Vertrauensverlust in der Finanzwelt. Die Unsicherheit über die Werthaltigkeit vieler Finanzprodukte erreichte ein zuvor nicht für möglich gehaltenes Ausmaß. In der Folge kam das Kreditgeschäft zwischen den Banken fast vollständig zum Erliegen. Soweit überhaupt noch kurzfristige Kredite gewährt wurden, mussten

21 Siehe die Pressemitteilung der Commerzbank AG vom 03. 11. 2008.

22 Siehe die Pressemitteilung der Commerzbank AG vom 08. 01. 2009.

23 Die Tagesordnung der Hauptversammlung und die Abstimmungsergebnisse sind abrufbar unter https:// www.commerzbank.de/de/hauptnavigation/aktionaere/haupt/hauptversammlung_1.html. Siehe ferner die Pressemitteilung der Commerzbank AG vom 05. 06. 2009.

24 Die Verschmelzung der Dresdner Bank auf die Commerzbank wurde am 11.05. 2009 ins Handelsregister eingetragen; siehe die Pressemitteilung der Commerzbank AG von diesem Tage.

25 Einer Dreiviertelmehrheit bedürfen Satzungsänderungen (§ 179 Abs. 1 und 2 AktG i. V.m. $§ 25$ Abs. 5 AktG) und Kapitalmaßnahmen (§ 182 Abs. 1, § 192 Abs. 1 i. V.m. $\S 193$ Abs. 1, 202 Abs. 1 und 2, 207 Abs. 1 und 2 i.V.m. $\S 182$ Abs. 1 und $\S 222$ Abs. 1 AktG).

26 Sachverständigenrat für die gesamtwirtschaftliche Entwicklung: Jahresgutachten 2007/08, 07. 11. 2008, Rn. 176 ff. (S. 122). 
sehr hohe Zinsen gezahlt werden. Demgegenüber blieben die Zinsen für längerfristige Staatsanleihen, die als ,sicherer Hafen“ angesehen wurden, relativ niedrig. Diese inverse Zinsstruktur belastete die Depfa und die HRE so erheblich, dass sie auf Staatshilfe angewiesen waren, um einen Zusammenbruch zu vermeiden. Umgekehrt war der Staat wegen der Systemrelevanz der HRE faktisch gezwungen, sie mit öffentlichen Mitteln zu stützen. ${ }^{27}$ Im März 2009 hatte die HRE einen Liquiditätsbedarf von 150 Mrd. $€$ und einen Eigenkapitalbedarf von 10 Mrd. $€$. Demgegenüber betrug die Marktkapitalisierung der HRE nur noch 190 Mio. $€$, also nicht einmal $2 \%$ des Bedarfs an Eigenkapital. ${ }^{28}$ Der Bund stellte in dieser Situation der HRE nicht nur einen Garantierahmen von 52 Mrd. $€$ zur Verfügung, sondern übernahm auch für mehrere Milliarden Euro sämtliche Anteile an der HRE, die Anfang Oktober 2009 einen negativen Unternehmenswert hatte und bei der zu diesem Zeitpunkt nach dem Gutachten einer großen Wirtschaftsprüfungsgesellschaft seinerzeit kein Szenario denkbar war, das zu einem positiven Wert der Aktien geführt hätte. ${ }^{29}$

Im November 2009 ist entschieden worden, dass der Bund der Westdeutschen Landesbank über den SoFFin mindestens 3 Mrd. $€$ zusätzliches Eigenkapital zur Verfügung stellen wird. Die Westdeutsche Landesbank wird mit einer Bilanzsumme von 260 Mrd. $€$ als systemrelevant angesehen. Zunächst wird die Westdeutsche Landesbank eine Abwicklungsbank, eine so genannte Bad Bank, gründen, der sie nicht mehr zum Kerngeschäft zahlende Aktiva in Höhe von 85 Mrd. $€$ einbringen wird. Der SoFFin wird dann der verbleibenden Kernbank das neue Eigenkapital zur Verfügung stellen. Es handelt sich um stille Einlagen, die später zum Teil oder vollständig in Stammkapital umgewandelt werden. ${ }^{30}$ Der Bund wird damit zum ersten Mal Gesellschafter einer Landesbank werden.

Der Bund ist folglich in Zukunft an drei weiteren bedeutenden deutschen Banken neben der Kreditanstalt für Wiederaufbau maßgeblich beteiligt, ohne dass er diese Beteiligungen angestrebt hätte. Das ändert aber nichts daran, dass er sich

27 So die übereinstimmende Einschätzung des Präsidenten der Deutschen Bundesbank, Prof. Dr. A. Weber, und des Präsidenten der BaFin, J. Sanio, Deutscher Bundestag, Finanzausschuss, Wortprotokoll, 120. Sitzung am 16.03. 2009, $3 \mathrm{f}$.

28 Präsident Sanio, J., a. a. O., 9, unter Bezug auf Äußerungen des früheren Vorsitzenden des SoFFin Dr. G. Merl.

29 PwC: Gutachterliche Stellungnahme zur Ermittlung der angemessenen Barabfindung der Aktien der Hypo Real Estate Holding AG, München, anlässlich der geplanten Übertragung der Minderheitsaktionäre gemäß § 327a Abs. 1 AktG i.V.m. § 12 Abs. 4 FMStBG zum Bewertungsstichtag 05.10. 2009, in: FMS, Übertragungsbericht, Anlage 1, S. 143 Rn. 542.

30 Frankfurter Allgemeine Zeitung v. 25.11. 2009, 1 und 13; Süddeutsche Zeitung v. 25.11. $2009,17$. 
als Gesellschafter nunmehr entscheiden muss, ob er seine Gestaltungsmöglichkeiten als Eigentümer zu einer Beeinflussung der Geschäftspolitik der jeweiligen Bank im Sinne seiner Definition des Gemeinwohls nutzen will oder ob er sich auf die Wahrung seiner Vermögensinteressen beschränkt und von einer Steuerung des Bankgeschäfts Abstand nimmt. Bevor der Antwort auf diese Frage nachgegangen wird, soll der Blick zunächst auf die Entwicklungen in Deutschland vergleichbaren ausländischen Staaten gerichtet werden.

\section{Die ausländischen Erfahrungen}

\section{Großbritannien}

Die Entwicklung in Großbritannien zwang den Staat noch stärker in die Rolle eines Aktionärs der letzten Instanz als der Verlauf der Finanzkrise in Deutschland. Auch im Vereinigten Königreich stiegen die Immobilienpreise in den Jahren vor dem Ausbruch der Krise steil an. In den fünf Jahren von 2002 bis 2007 verdoppelten sich die Immobilienpreise beinahe. Im Verlaufe dieser ,house price bubble" wurde die so genannte ,property ladder" äußerst populär: Nur kurze Zeit nach dem Erwerb eines kleineren Hauses (regelmäßig auf Kredit) wurde dieses mit solchem Gewinn veräußert, dass ein größeres Haus erworben werden konnte. Dieses wurde bald erneut mit Gewinn veräußert und ein wiederum größeres Haus erworben. Solange die Hauspreise stiegen, versprach ein derartiges Vorgehen hohen Gewinn. Als aber nach dem Platzen der Spekulationsblase die Hauspreise fast ebenso schnell wieder sanken, wie sie zuvor gestiegen waren, erlitten die Eigentümer nicht nur große Verluste, sondern gerieten auch die Hypothekenkredite in Gefahr. So geriet zunächst der größte Baufinanzierer Großbritanniens Northern Rock in große wirtschaftliche Schwierigkeiten und musste die Bank von England um ein Notdarlehen bitten. Als diese Darlehensgewährung bekannt geworden war, begann am 14. September 2008 ein so genannter ,bank run“. Innerhalb von zwei Tagen hoben die Kunden der Bank Einlagen im Wert von über zwei Milliarden ab. Die britische Regierung sah sich darauf hin gezwungen, eine staatliche Garantie für alle Bankeinlagen abzugeben. Sie versuchte zunächst, Northern Rock an private Investoren zu veräußern, erhielt aber kein zufriedenstellendes Angebot. Mit Blick auf die $55 \mathrm{Mrd}$ £, die sie der Bank bereits an Garantien und Bürgschaften gegeben hatte, entschloss sie 
sich am 17. Februar 2008 zur Verstaatlichung. ${ }^{31}$ Die Rechtsgrundlage für die Verstaatlichung wurde hastig mit dem Banking (Special Provisions) Act 2008 geschaffen, der bereits am 21. Februar in Kraft trat. ${ }^{32}$ Er war auf ein Jahr befristet und wurde durch den Banking Act $2009^{33}$ ersetzt. Auf der neu geschaffenen gesetzlichen Grundlage verstaatlichte die britische Regierung Northern Rock. Eine Entschädigung für die Gesellschafter der Bank wurde nicht gewährt, weil die Regierung die Aktien für wertlos hielt. ${ }^{34}$ Die Eigentümerbefugnisse nimmt die zur Verwaltung der britischen Staatsbeteiligungen gegründete UK Financial Investments Ltd. wahr. ${ }^{35}$ Nur sieben Monate später sah sich die britische Regierung gezwungen, erneut von den ihr durch den Banking (Special Provisions) Act 2008 eröffneten Möglichkeiten Gebrauch zu machen: Sie verstaatliche am 29. September 2008 das Hypothekengeschäft der Bank Bradford \& Bingley, der als Folge der Finanzkrise ebenfalls der Zusammenbruch drohte. ${ }^{36}$ Der britische Staat führt auch die Geschäfte dieser Bank über die Holdinggesellschaft UK Financial Investments Ltd. ${ }^{37}$

Nur neun Tage später musste die britische Regierung am 8. Oktober ein Bankenrettungspaket mit einem Umfang von 500 Mrd. $£$ auflegen. ${ }^{38}$ Ein wichtiger Bestandteil des Pakets ist der Bank Recapitalisation Fund, der mit 50 Mrd. $£$ ausgestattet ist. Für die Kapitalbeteiligung erhält der Staat sowohl Stamm- als auch Vorzugsaktien, mit denen kein Stimmrecht verbunden ist. Die Vorzugsaktien sind nicht dividendenberechtigt. Stattdessen wird das durch sie repräsentierte Kapital mit einem festen Satz verzinst. Sowohl die Höhe der jeweiligen Staatsbeteiligung als auch die Verteilung von Stamm- und Vorzugsaktien wird in Verhandlungen zwischen dem Staat und den betroffenen Banken festgelegt. Aus den Mitteln des Fund rekapitalisierte der Staat die Royal Bank of Scotland mit 20 Mrd. £. Davon entfielen 15 Mrd. £ auf stimmrechtsberechtigte Stammaktien und

$31 \mathrm{http} / / /$ business.timesonline.co.uk/tol/business/industry_sectors/banking_and_finance/article3385758.ece.

$32 \mathrm{http}: / /$ www.statutelaw.gov.uk/content.aspx?activeTextDocId=3450001.

33 http://www.opsi.gov.uk/acts/acts2009/ukpga_20090001_en_1.

34 http://news.sky.com/skynews/Home/Business/Northern-Rock-Shareholders-Lose-Court-Case-Governme nt-Did-Not-Infringe-Human-Rights/Article/200902215222493.

35 Siehe die Pressemitteilung 114/08 des HM Treasury vom 03.11. 2008. Die Pressemitteilungen des HM Treasury sind abrufbar unter http://www.hm-treasury.gov.uk/press_notices_index.htm.

36 Die spanische Bank Grupo Santander übernahm nicht nur das Filialnetz, sondern auch die Spareinlagen von Bradford \& Bingley. Siehe die Pressemitteilung 97/08 des HM Treasury vom 29. 09. 2008.

37 Siehe die Pressemitteilung 114/08 des HM Treasury vom 03.11. 2008.

38 Siehe die Pressemitteilung 100/08 des HM Treasury vom 08. 10. 2008. 
5 Milliarden $£$ auf Vorzugsaktien. ${ }^{39}$ Nach dieser Rekapitalisierung hält der britische Staat mit $58 \%$ die Mehrheit an der Bank. Nachdem zunächst vereinbart worden war, die $5 \mathrm{Mrd} £$ mit $12 \% \mathrm{zu}$ verzinsen, stellte sich bald heraus, dass die Bank die daraus erwachsende Zinslast von jährlich 600 Mio. £ nicht tragen konnte. Deshalb wurden die Vorzugsaktien im Januar 2009 in Stammaktien umgewandelt. ${ }^{40}$ Seitdem beträgt der Anteil der Regierung an der Royal Bank of Scotland ungefähr $70 \%{ }^{41}$

Bei der Halifax Bank of Scotland verlief die Entwicklung etwas anders: Als sie in eine bedrohliche Schieflage geriet, wurde sie von der Bank Lloyds TSB übernommen. Diese Übernahme brachte jedoch Lloyds TSB in so große Schwierigkeiten, dass ein Eingriff des Staates unausweichlich wurde. Er beteiligte sich mit 17 Mrd. $£$ an der Bank und erhielt dafür Stammaktien im Wert von 13 Mrd. $£$ sowie Vorzugsaktien von 4 Mrd. . $^{42}$ Die Verzinsung der Vorzugsaktien in Höhe von $12 \%$ brachte eine jährliche Zinslast von 480 Mio. $£$ mit sich, die sich für Lloyds TSB bald als zu hoch erwies. Daraufhin wurden im März 2009 auch bei dieser Bank die Vorzugsaktien des Staates in Stammaktien umgewandelt. ${ }^{43}$ Auch die Beteiligungen des britischen Staates an der Royal Bank of Scotland und an Lloyds TSB liegen in der Hand der UK Financial Investments Ltd., die alle Gesellschafterrechte des Staates wahrnimmt. ${ }^{44}$ Der Staat will bei den Banken, an denen er sich beteiligt hat, im operativen Geschäft Einfluss nehmen. Insbesondere Bonuszahlungen sollen stark eingeschränkt und Dividendenzahlungen untersagt werden. ${ }^{45}$

\section{Vereinigte Staaten von Amerika}

Nachdem in den Vereinigten Staaten von Amerika zunächst geplant war, aus den Mitteln eines Rettungsfonds über $700 \mathrm{Mrd}$. \$ den Banken risikobehaftete Wertpapiere abzukaufen, begründete der Emergency Economic Stabilization Act im

39 http://news.bbc.co.uk/2/hi/business/7666570.stm.

40 Siehe die Pressemitteilung 06/09 des HM Treasury vom 19.01. 2009.

$41 \mathrm{http}: / /$ newsvote.bbc.co.uk/2/hi/business/7836882.stm.

42 http://news.bbc.co.uk/2/hi/business/7666570.stm.

43 http://www.telegraph.co.uk/finance/newsbysector/banksandfinance/4796477/Lloyds-in-lending-deal-wit h-Alistair-Darling.html.

44 Siehe die Pressemitteilung der UK Financial Investments Limited vom 01.12. 2008, abrufbar unter http://www.ukfi.gov.uk/releases/ukfi_takes_on_mngmnt_rbs_shares.pdf.

45 Sachverständigenrat zur Begutachtung der gesamtwirtschaftlichen Entwicklung: Die Finanzkrise meistern - Wachstumskräfte stärken. Jahresgutachten 2008/09 vom 12.11. 2008, 154. 
Oktober 2008 das Troubled Assets Relief Program (TARP). Das Finanzministerium der USA verwaltet das TARP über das neu eingerichtete Office of Financial Stability. Die Regierung erzwang auf dieser Grundlage eine Beteiligung an neun Großbanken: Bank of America, JPMorgan, Wells Fargo, Citigroup, Merrill Lynch, Goldman Sachs, Morgan Stanley, Bank of New York Mellon und State Street. Die Banken wurden mit bis zu 25 Mrd. \$ rekapitalisiert. Für diese Maßnahmen stehen insgesamt bis zu $250 \mathrm{Mrd}$. \$ zur Verfügung. Im Gegenzug wurden stimmrechtslose Vorzugsaktien ausgegeben. Sie erhalten in den ersten fünf Jahren eine Dividende von $5 \%$, die sodann auf $9 \%$ steigen soll. Der Staat erhält zudem neben den Aktien Optionsscheine, die zum Erwerb von Aktien berechtigen. So soll der Staat an künftigen Kursgewinnen beteiligt werden. Erwirbt er aus den Mitteln des TARP Vermögenswerte der Banken, die wegen der Krise an Wert verloren haben, erhält er zum Ausgleich ebenfalls Optionsscheine, die ihm zukünftig eine Beteiligung an den Banken und damit auch an ihren Gewinnen ermöglichen. ${ }^{46}$ Der Staat beteiligt sich damit in den USA weit stärker als in Europa an systemrelevanten Banken und hat auf einen möglichen entgegenstehenden Willen der betroffenen Unternehmen keine Rücksicht genommen.

\section{Die Handlungsalternativen}

Nicht nur in Deutschland, sondern auch in Großbritannien und den USA ist der Staat also als Folge seiner Beistandsleistungen für von der Insolvenz bedrohte Banken zum Bankier geworden. Er ist alleiniger oder jedenfalls maßgeblicher Gesellschafter systemrelevanter Finanzinstitute geworden, ohne dass er diese Stellung angestrebt oder auch nur als wünschenswert angesehen hätte. Am Ergebnis ändert das nichts. Deshalb stehen alle betroffenen Staaten nun vor dem Problem, wie sie sich als Bankgesellschafter verhalten sollen. Eine Alternative wäre die Rolle des temporären stillen Gesellschafters: Der Staat verzichtet darauf, auf die Geschäftspolitik seiner Unternehmen Einfluss zu nehmen und bemüht sich darum, seine Gesellschaftsanteile so schnell wie möglich wieder Privaten $\mathrm{zu}$ übertragen. Er müsste allerdings damit rechnen, bei einer erneuten Bankenkrise wieder als Aktionär der letzten Instanz in Anspruch genommen zu werden. Er müsste dann erneut öffentliche Gelder einsetzen, um eine Insolvenz von Banken zu verhindern, die als systemrelevant gelten. Maßnahmen gegen das Entstehen solcher Schieflagen könnte er nur von außen als Regulator treffen ein Bemühen, das allerdings bei der aktuellen Finanzkrise praktisch vollständig 
fehlgeschlagen ist. ${ }^{47}$ Zudem würde er in der Rolle des temporären stillen Gesellschafters darauf verzichten, seine Politikziele wie die Verhinderung einer Kreditklemme, die Begrenzung der Gehälter und Boni von Bankmanagern oder die Neuordnung der Landesbanken aus seiner Gesellschafterstellung heraus zu befördern.

Das Gegenmodell bestünde darin, dass der zum Bankier gewordene Staat seine Beteiligungen als grundsätzlich dauerhaft betrachtete und aktiv auf die Geschäftspolitik seiner Unternehmen einwirkte, um sie im Sinne des von ihm definierten Gemeinwohls zu beeinflussen. Als Gesellschafter nähme er Unternehmerbefugnisse wahr und brauchte sein Handeln nicht als Eingriff in die unternehmerische Freiheit Dritter zu rechtfertigen. Er stünde allerdings vor der Notwendigkeit, sich des erforderlichen bankfachlichen Sachverstands von außen versichern zu müssen, weil kaum zu erwarten sein würde, dass hinreichend qualifizierte Manager in der öffentlichen Verwaltung zu finden wären. Zugleich trüge er die Risiken einer verfehlten Geschäftspolitik, wie sie ganz offensichtlich viele Landesbanken in den vergangenen Jahren verfolgt haben. Der Staat ist kein besserer Bankier als private Unternehmer. Angesichts der finanziellen Schwierigkeiten bedeutender privater Banken wie der Dresdner Bank, der Commerzbank, der HRE oder des Privatbankhauses Sal. Oppenheimer scheint allerdings auch die Behauptung gewagt, private Unternehmer und Manager seien erfolgreicher und vermieden Fehleinschätzungen besser als ihre Widerparts im Bereich des öffentlichen Bankwesens. An Negativbeispielen herrscht sowohl im Bereich des öffentlichen wie des privaten Bankwesens kein Mangel. Eine dauerhafte Beteiligung böte dem Staat zudem den Vorteil, dass er auch längerfristig an den Gewinnen seiner Banken beteiligt wäre und auf diesem Wege einen Teil der Gelder zurückerhielte, mit denen er in der Finanzkrise die in eine Schieflage geratenen Banken unterstützt hat. Sozialisiert würden nicht nur die Verluste, sondern auch die Gewinne. Mildern würde sich auch der Druck in Richtung auf eine Entflechtung systemrelevanter Banken, wie sie vor allem in den USA, aber auch in Deutschland nach dem Satz "If they are too big to fail, they are too big “ in Erwägung gezogen, von den betroffenen Banken aber entschieden bekämpft wird.

Denkbar sind auch Zwischenlösungen: So könnte der Staat sich zeitweilig als aktiver Gesellschafter verstehen und bis zur Bewältigung der Finanzkrise von seinen Gesellschafterbefugnissen Gebrauch machen, um seine Ziele wie die 
Sicherstellung eines angemessenen Kreditangebots für die Wirtschaft, die Verhinderung verfehlter Anreize für Bankmanager, überhöhte Risiken einzugehen und die Neuordnung der Landesbanken zu verfolgen. Umgekehrt könnte er sich auch von einer aktiven Geschäftspolitik fernhalten, aber seine Beteiligungen als dauerhafte Anlagen mit dem Ziel behandeln, langfristig zumindest einen Teil der Schulden, die er zur Stützung gefährdeter Banken in der Finanzkrise aufgenommen hat, aus den erhofften zukünftigen Gewinnen der geretteten Banken zu begleichen.

\section{Die normative Ordnung}

\section{Die Verfassungsvorgaben}

Aus rechtlicher Sicht stellt sich die Frage, ob es normative Vorgaben für die Wahl zwischen den aufgezeigten Alternativen gibt. Zunächst ist insoweit die Verfassung in den Blick zu nehmen. Der Streit, ob das Grundgesetz zumindest implizit eine Wirtschaftsverfassung enthält, ist seit mehr als fünfzig Jahren dahin entschieden. Bereits 1954 hat das Bundesverfassungsgericht entschieden, dass das Grundgesetz „weder die wirtschaftspolitische Neutralität der Regierungsund Gesetzgebungsgewalt noch eine nur mit marktkonformen Mitteln zu steuernde ,soziale Marktwirtschaft““ garantiere. Die „wirtschaftpolitische Neutralität" des Grundgesetzes bestehe nach der Rechtsprechung des Bundesverfassungsgerichts darin, dass sich der Verfassungsgeber nicht ausdrücklich für ein bestimmtes Wirtschaftssystem entschieden habe. So sei es dem Gesetzgeber möglich, ,die ihm jeweils sachgemäß erscheinende Wirtschaftspolitik zu verfolgen, sofern er dabei das Grundgesetz beachtet.“ Der Staat müsse bei seinem wirtschaftlichen Handeln also zwar die Grundrechte beachten, ihm sei aber von Verfassungs wegen eine bestimmte Ordnung der Wirtschaft nicht vorgegeben. ${ }^{48}$ Das Grundgesetz steht also einer wirtschaftlichen Betätigung des Staates auch im Bereich des Bankwesens nicht entgegen. Es ist verfassungsrechtlich nicht vorgegeben, sondern steht im politischen Ermessen der demokratisch legitimierten Amtswalter, ob der Staat bloßer Aktionär der letzten Instanz bleibt oder sich in

48 BVerfGE 4, 7 ff., hier 17 f.; BVerfGE 50, 290 ff., hier $336 \mathrm{ff}$.; aus der Literatur einerseits Hellermann, J.: Örtliche Daseinsvorsorge und gemeindliche Selbstverwaltung, Tübingen, 2000, $161 \mathrm{ff}$., und andererseits Rupp, H.: Die Soziale Marktwirtschaft in ihrer Verfassungsbedeutung, in: Isensee, J./Kirchhof, P. (Hg.): Handbuch des Staatsrechts der Bundesrepublik Deutschland, Bd. IX, Heidelberg, 1997, § 203 Rn. $16 \mathrm{ff}$. m.w.N. 
der ihm aufgedrängten Rolle als Bankier einrichtet und deren Chancen und Risiken annimmt.

Von Bedeutung ist insoweit allerdings auch das Prinzip der Wirtschaftlichkeit, das eine Optimierung des Ertrags vom Staat eingesetzter öffentlicher Mittel gebietet (Art. 114 Abs. 2 Satz 1 GG). ${ }^{49}$ Der Staat hat beträchtliche Milliarden zur Stützung von der Insolvenz bedrohter systemrelevanter Banken aufgewendet und seine Verschuldung entsprechend erhöht. Das Prinzip der Wirtschaftlichkeit verpflichtet ihn nun, die auf diesem Wege erworbenen Beteiligungen an Finanzinstituten so zu behandeln, dass er für seine enormen finanziellen Aufwendungen einen möglichst hohen Gegenwert erhält. Dieser Gegenwert muss nicht in Geld bestehen. Der Staat ist aber durch das Wirtschaftlichkeitsprinzip gehindert, auf das von ihm eingesetzte öffentliche Vermögen ohne angemessene Gegenleistung zu verzichten. Das dürfte ihm jedenfalls gegen eine Veräußerung der erworbenen Unternehmensbeteiligungen unter dem Einstandspreis im Sinne der von ihm insgesamt zur Stützung des jeweiligen Bankunternehmens aufgewandten Mittel verbieten.

\section{Die Europarechtsvorgaben}

Ebenso wenig wie das Verfassungsrecht steht das Europarecht dem staatlichen Eigentum an Banken entgegen. Art. 295 EGV lässt die Eigentumsordnung in den verschiedenen Mitgliedstaaten ausdrücklich unberührt. Damit ist insbesondere das Recht der Mitgliedstaaten zur Verstaatlichung und sogar Enteignung privater Vermögenswerte durch das Gemeinschaftsrecht unberührt, soweit der Grundsatz der Nichtdiskriminierung beachtet wird. ${ }^{50}$ Die Wirtschaftspolitik der Mitgliedstaaten und der Gemeinschaft ist dem Grundsatz einer offenen Marktwirtschaft mit freiem Wettbewerb verpflichtet (Art. 4 Abs. 1 EGV). ${ }^{51}$ Sie erkennt aber

49 Näher Heun, W., in: Dreier, H. (Hg.): Grundgesetz. Kommentar, Bd. III, 2. Aufl., Tübingen, 2008, Art. 114 Rn. 29.

50 EuGH, Urt. v. 06.11. 1984, Rs. 182/83 (Fearon/Irish Land Commission), Slg. 1984, 3677, hier 3685, Rn. 7; vgl. auch EuGH, Urt. v. 04.06. 2002, Rs. $367 / 98$ (Kommission/Portugal), Slg. 2002, I-4731, hier 4774, Rn. 48; EuGH, Urt. v. 04.06. 2002, Rs. $483 / 99$ (Kommission/Frankreich), Slg. 2002, I-4781, hier 4803, Rn. 44; EuGH, Urt. v. 04.06. 2002, Rs. 503/99 (Kommission/Belgien), Slg. 2002, I-4809, hier 4832, Rn. 44; EuGH, Urt. v. 13.05. 2003, Rs. 463/00 (Kommission/Spanien), Slg. 2003, I-4581, hier 4633, Rn. 67; aus der Literatur Schweitzer, M., in: Grabitz, E./Hilf, M. (Hg.): Das Recht der Europäischen Union. Kommentar, München, Loseblatt, 2005, Art. 295 EGV Rn. 4 ff.

51 Dazu Bandilla, R., in: Grabitz, E./Hilf, M. (Hg.): Das Recht der Europäischen Union, München, Loseblatt, 2000, Art. 4 EGV Rn. 5 ff. 
öffentliche Unternehmen ausdrücklich an (Art. 86 Abs. $1 \mathrm{GG})^{52}$ und hebt die Bedeutung der Dienste von allgemeinem wirtschaftlichen Interesse nachdrücklich hervor (Art. 16 EGV) ${ }^{53}$. Damit steht aus gemeinschaftsrechtlicher Sicht einer Tätigkeit des Staates als Bankunternehmer weder in der Zeit der Finanz- und Wirtschaftskrise noch in der Zeit nach deren Überwindung etwas entgegen.

\section{Die Folgerungen}

Welche Folgerungen soll der Staat aus der Finanz- und Wirtschaftskrise ziehen? Der zunächst auf den wenigsten Widerstand stoßende Weg dürfte es sein, weiterhin auf die Regulierung des Finanzsystems zu vertrauen und die in der Krise gegen den Willen der staatlichen Akteure erworbenen Beteiligungen an Finanzunternehmen so schnell wie möglich wieder zu privatisieren. Dann droht dem Staat bei einer erneuten Finanz- und Wirtschaftskrise das Schicksal eines Aktionärs letzter Instanz, wie es vom Vorstandsvorsitzenden der Deutschen Bank Ackermann in Aussicht gestellt wurde. Er müsste wiederum Garantien aussprechen und Beteiligungen erwerben, wenn systemrelevante Banken vom Zusammenbruch bedroht wären. Das wäre erneut mit dem Einsatz öffentlicher Gelder in mehrstelliger Milliardenhöhe und aller Voraussicht nach mit einer weiteren Erhöhung der Staatsverschuldung verbunden. Dann stellte sich die Frage, ob ein solches Vorgehen den Bürgerinnen und Bürgern noch zu vermitteln wäre. Die Antwort auf diese Frage würde auch nicht durch die Einrichtung des von Seiten der Banken vorgeschlagenen Fonds leichter, in den schon vor einer Krise nicht nur Beiträge der Banken, sondern auch beträchtliche öffentliche Gelder eingezahlt werden müssten, um in einer neuen Krise zu einem geordneten Verfahren der Bankenstützung zu gelangen. Der Bevölkerung dürfte zu Recht kaum zu erklären sein, warum der Staat gewissermaßen zu Stützungsmaßnahmen auf Vorsorge greifen sollte, wenn eine Bedrohung des Finanzsystems nur eine abstrakte Gefahr darstellt, die sich noch nicht konkretisiert hat. Es käme auch zu dem Problem, dass in der marktwirtschaftlichen Ordnung zukünftig manche Marktteilnehmer ohne das Risiko eines Fehlschlagens ihrer Bemühungen agieren könnten, weil sie systemrelevant für das Finanzsystem wären. Das würde die vom Staat normierten Rahmenbedingungen der sozialen Marktwirtschaft grund-

52 Dazu Pernice, I./Wernicke, S., in: Grabitz, E./Hilf, M. (Hg.): Das Recht der Europäischen Union, München, Loseblatt, 2003, Art. 86 EGV Rn. 20.

53 Dazu Pernice, I./Wernicke, S., in: Grabitz, E./Hilf, M. (Hg.): Das Recht der Europäischen Union, München, Loseblatt, 2003, Art. 16 EGV Rn. 1 ff. 
legend ändern und zugleich die Gefahr des moral hazard deutlich verschärfen. Warum sollte ein Unternehmen Geschäfte meiden, die einen hohen Gewinn bei hohem Risiko versprechen, wenn die Allgemeinheit die Risikobewältigung ganz oder jedenfalls zu einem beträchtlichen Teil übernimmt?

Ein anderes Vorgehen würde bei der Systemrelevanz von Banken ansetzen. Deren volkswirtschaftliche Risiken ließen sich verringern, wenn auch vielleicht nicht ganz beseitigen, wenn systemrelevante Finanzinstitute vom Staat zur Entflechtung gezwungen würden. Dadurch könnte die Zahl systemrelevanter Unternehmen verringert werden. Ein solches Vorhaben könnte jedoch von einem einzelnen Staat kaum durchgesetzt werden, weil sich die betroffenen Banken dessen entflechtendem Zugriff durch eine Sitzverlagerung in einen anderen Staat entziehen könnten. Ob aber ein Zusammenwirken der potentiell betroffenen Staaten bei der Entflechtung von systemrelevanten Banken zu organisieren ist, muss wegen der sehr unterschiedlichen Interessen und Vorstellungen der handelnden politischen Akteure und wegen divergierender nationaler Wirtschaftsinteressen bezweifelt werden.

Kommt es aber zu keiner Entflechtung systemrelevanter Banken und bleibt damit die gegenwärtige Lage, dass bestimmte Finanzinstitute wegen ihrer Größe im Falle einer drohenden Insolvenz gerettet werden müssen, auch in Zukunft unverändert bestehen, muss der Staat die Option, nicht nur von außen regulierend tätig zu werden, sondern kraft der ihm zugewachsenen Unternehmensbeteiligungen von innen auf die Geschäftspolitik großer Banken dort einzuwirken, wo ihm das kraft seiner Gesellschafterstellung möglich ist, zumindest in Erwägung ziehen. Auch als Gesellschafter eines Finanzunternehmens erfüllt der Staat eine Verwaltungsaufgabe. Seine Aufgabe der Unternehmensträgerschaft setzt einen öffentlichen Zweck voraus. ${ }^{54}$ Dieser öffentliche Zweck besteht bei der hier in Rede stehenden Beteiligung an systemrelevanten Banken in der Stabilisierung des Finanzsystems. Zur Zweckerreichung kann ein Beibehalten der Gesellschafterstellung erforderlich sein, wenn die regulatorischen Einwirkungsmöglichkeiten nach den Einschätzungen der für den Staat handelnden Akteure nicht sicher genug ausreichen, um eine neue Finanzkrise zu verhindern.

In welchem Umfang sich der Staat aus seiner Gesellschafterstellung heraus unternehmerisch betätigen sollte, ist eine andere Frage. Insoweit kann es durchaus sinnvoll sein, sich im Wesentlichen auf die Wahrung der staatlichen Vermögens-

54 Osterloh L.: Privatisierung von Verwaltungsaufgaben, in: Veröffentlichungen der Vereinigung der Deutschen Staatsrechtslehrer, 54 (1995), 204-242, hier $227 \mathrm{f}$. 
interessen zu beschränken. Das betrifft sowohl Garantien und Bürgschaften als auch die Steigerung des Werts der Beteiligung des Staates. Will der Staat als Unternehmensträger andere öffentliche Zwecke wie etwa das Vermeiden einer Kreditklemme verfolgen, muss er sich darüber klar werden, in welchem Umfang ein solches Vorgehen dem Zweck der Sicherung eigener Vermögensinteressen und der Stabilisierung der Finanzmärkte entgegen läuft und wie ein sachgerechter Ausgleich gefunden werden kann. Ein derartiges dauerhaftes Engagement ginge deutlich über die Rolle eines Aktionärs der letzten Instanz hinaus, die Ackermann für den Staat im Bankbereich für angezeigt hält. Sie würde aber besser der staatlichen Verantwortung für die wirtschaftliche Verwendung öffentlicher Gelder gerecht als ein bloßes Eingreifen zur Abwehr eines unmittelbar bevorstehenden Zusammenbruchs eines Finanzinstituts in einer Krisensituation. Ein solches Vorgehen kostete den Staat nur viel Geld, ohne dass ein entsprechender finanzieller Ausgleich in absehbarer Zeit gesichert wäre. 